\title{
Características epidemiológicas del paciente que ingresa en la Unidad de Hospitalización Psiquiátrica del Departamento de Salud de Gandía
}

\author{
Epidemiological Characteristics of the Patient Admitted to the Psychiatric Hospitalization Unit
} of the Gandía Health Department

\author{
Pilar Palomares Díaz, Mercedes Rodríguez Martí, Carlos Vercher Félix \\ Unida de Hospitalización Psiquiátrica del Hospital Francesc de Borja de Gandía. Gandía, España. \\ Contacto: pipadiaz@gmail.com \\ Fecha de recepción: 8 de abril de 2017 / Fecha de aceptación: 25 de junio de 2017
}

\begin{abstract}
Resumen
Introducción: Se analiza el perfil sociodemográfico y clínico de los pacientes que acuden a la Unidad de Hospitalización Psiquiátrica del Hospital Francesc de Borja de Gandía.

Objetivos: Analizar variables sociodemográficas y clínicas de los pacientes que fueron ingresados en la UHP desde el 1 de enero de 2011 hasta el 31 de diciembre de 2013.

Metodología: Estudio descriptivo retrospectivo de los pacientes que ingresan en la UHP con un número total de participantes de n=210 en 2011, n=206 en 2012 yn=247 en 2013, según los datos extraídos del CMBD de dicho hospital.

Resultados: En el año 2011, el perfil del paciente ingresado fue: hombre (55,71\%), de entre 41 y 50 años (29,05\%), cuyo motivo de ingreso es descompensación psicótica (36,67\%) y con diagnóstico principal esquizofrenia $(24,76 \%)$ que permanece en la unidad entre 8 y 14 días (26,19\%). Por otro lado, en el año 2012, el perfil quedaría de la siguiente forma: hombre (50,97\%), de entre 41 y 50 años (31,55\%), cuyo motivo de ingreso es descompensación psicótica $(45,15 \%)$ y con diagnóstico principal de trastorno bipolar $(23,30 \%)$ que permanece en la unidad de 8 a 14 días $(24,76 \%)$. En cuanto al 2013 , observamos que el perfil sigue la misma línea del año anterior siendo en este año: hombre (52,63\%), de 31 a 40 años (27,53\%), cuyo motivo de ingreso es descompensación psicótica (37,65\%) y con diagnóstico principal de trastorno bipolar (27,13\%) que permanece en la unidad de 8 a 14 días (23,48\%).

Conclusiones: Tras el análisis de los datos, apreciamos que el perfil del paciente que ingresa en nuestra unidad no difiere del resto de estudios publicados: varón (apenas se aprecia variación en cuanto a sexo), de edad media, con diagnóstico trastorno psicótico y una estancia aproximada de 15-30 días ${ }^{1-4}$.
\end{abstract}

Palabras clave: perfil sociodemográfico, Unidad de Hospitalización psiquiátrica, departamento de Gandía.

\begin{abstract}
Introduction: The sociodemographic and clinical profile of the patients who come to the Psychiatric Hospitalization Unit of the Francesc de Borja Hospital in Gandia is analyzed.

Objectives: To analyze sociodemographic and clinical variables of the patients who were admitted to the UHP from January 1, 2011 to December 31, 2013.

Methodology: Retrospective descriptive study of patients admitted to the UHP with a total number of participants of n=210 in 2011, n=206 in 2012 and n=247 in 2013, according to the data extracted from the CMBD of said hospital. Results: In 2011, the profile of the admitted patient was: male (55.71\%), between 41 and 50 years old (29.05\%), whose reason for admission is psychotic decompensation (36.67\%) and with main diagnosis schizophrenia $(24.76 \%)$ that remains in the unit between 8 and 14 days (26.19\%). On the other hand, in 2012 the profile would be as follows: man (50.97\%), between 41 and 50 years (31.55\%), whose reason for admission is psychotic decompensation (45.15\%) and with a main diagnosis of bipolar disorder (23.30\%) that remains in the unit for 8 to 14 days (24.76\%). As for 2013 , we observe that the profile follows the same line of the previous year being this year: male (52.63\%), from 31 to 40 years old $(27.53 \%)$, whose reason for admission is psychotic decompensation $(37,65 \%)$ and with a main diagnosis of bipolar disorder $(27.13 \%)$ that remains in the unit for 8 to 14 days $(23.48 \%)$.
\end{abstract}


Conclusions: After the analysis of the data, we can see that the profile of the patient admitted to our unit does not differ from the rest of the published studies: male (little variation in sex), average age, with a psychotic disorder diagnosis and an approximate stay $15-30$ days $^{1-4}$.

Keywords: sociodemographic profile, psychiatric hospitalization unit, department of Gandía.

\section{Introducción}

Los trastornos mentales constituyen una patología que se presenta en todas las culturas, apareciendo a cualquier edad. En términos absolutos, se estima que cada año 450 millones de personas en el mundo van a sufrir algún problema que podría catalogarse de trastorno mental o de conducta; constituyen, por tanto, una serie de procesos patológicos que requieren asistencia sanitaria especializada ${ }^{1-5}$. Este departamento cuenta con distintos recursos para la atención psiquiátrica: Unidad de Salud Mental, Hospital de día, Hospitalización domiciliaria específica de Salud Mental, Unidad de Hospitalización de Salud Mental, Centros de Rehabilitación (CRIS y Centro de Día), pisos tutelados, Col·lectiu Obertament.

Los pacientes con enfermedad mental grave presentan una gran disparidad en su evolución siendo común el mayor o menor deterioro en distintos aspectos de su personalidad determinando en la mayoría una dificultad más o menos importante para ejercer su propia autonomía. Toda esta situación va a repercutir sobre las familias, quienes deben verse cada vez más involucradas en los distintos tratamientos, y a la vez, sobre la sociedad debiendo ser ésta depositaria de mensajes para poder aceptar mejor al enfermo (estigmatización).

A pesar de todos los recursos existentes en el departamento, dada la evolución en ocasiones tórpida de la enfermedad, es necesario el ingreso en la unidad de agudos, entendida ésta como un elemento más dentro del plan de cuidados general del enfermo; su mayor preparación y colaboración en este plan de cuidados va a ser determinante para ocupar un papel relevante dentro de los recursos mencionados.

Por todo lo anterior, se considera fundamental que la UHP conozca las características de la población a la que atiende con el fin de planificar la atención, docencia e investigación adecuadas. El estudio de las características sociodemográficas y epidemiológicas de los pacientes que requieren ingreso en la UHP del Hospital Comarcal Francesc de Borja de Gandía puede permitir detectar factores que influyan en la evolución de los pacientes, así como mejorar la calidad asistencial de esta unidad. Es por ello que el objetivo general del estudio es describir el perfil del paciente que ingresa en la UHP del Departamento de Salud de Gandía en los años arriba mencionados. Por este motivo, los objetivos específicos se centran en: estudiar el perfil demográfico por sexo, edad y diagnóstico; describir los diagnósticos según la frecuencia de ingreso en la UHP; estimar la estancia media de ingreso; relacionar la estancia media de ingreso por patologías; exponer la evolución temporal anual de los ingresos.

\section{Metodología}

Estudio epidemiológico descriptivo retrospectivo de los pacientes que han precisado ingreso en la Unidad de Hospitalización Psiquiátrica del Hospital Francesc de Borja de Gandía durante el periodo comprendido entre el 1 de enero del 2011 hasta el 31 de diciembre del 2013.

Sujeto a estudio. Forman parte de este estudio todos aquellos pacientes que han ingresado en la Unidad de Hospitalización Psiquiátrica del hospital Francesc de Borja de Gandía durante los años 2011, 2012, 2013.

Zona de estudio. Este estudio se centra en la Unidad de Hospitalización Psiquiátrica del Hospital Francesc de Borja, hospital de referencia del departamento de Gandía, que presta asistencia a más de 188000 habitantes de las 31 poblaciones de la comarca de la Safor y de diez municipios de La Vall d'Albaida.

Se trata de una unidad que dispone de 9 camas para ingreso de pacientes con patología psiquiátrica. La característica principal de la sala es que se trata de una unidad abierta donde también se encuentran pacientes de medicina interna. Otra de las características de esta sala es que comparte ubicación con el Hospital de día de enfermos de salud mental; y aunque no comparten personal sanitario, sí comparte alguna de sus instalaciones. En cuanto al personal que atiende a la unidad, la componen una psiquiatra, 13 enfermeros y 9 auxiliares 
de enfermería. Tanto enfermería como auxiliares rotan periódicamente entre los dos servicios, de manera que la atención de dicho personal a este servicio UHP constará de una enfermera y una auxiliar por turno (mañana, tarde y noche).

Variables. En cuanto a las variables, se van a utilizar aquellas que nos proporciona el programa CMBD de hospitalización que nos aporta el servicio de admisión del Hospital Francesc de Borja. Las variables a estudiar van a ser: edad (en años); sexo: (hombre/mujer); número total de ingresos; motivo de ingreso; estancia media: obtenida de la diferencia entre la fecha de alta y la de ingreso que vendrá expresada en número de días; diagnóstico. Para respetar la protección de datos del paciente, el servicio de admisión del hospital Francesc de Borja de Gandía proporcionará los datos disociativos que no incluyen variables identificativas de los pacientes participantes en el estudio.

Fuente y recogida de datos. Los datos que se van a utilizar en este estudio los proporciona la base de datos de admisión del hospital Francesc de Borja (CMBD de hospitalización). De ésta podemos recopilar los siguientes datos: fecha de nacimiento y sexo.

En cuanto a los datos de identificación del episodio que se incorpora al CMBD a través del registro de hospitalización así como los registros de la UCSI (unidad de admisión) tanto en el momento del ingreso como al alta hospitalaria. Los datos a utilizar de este punto van a ser el número de ingresos o asistencia, que los proporciona automáticamente el programa, la fecha de ingreso, el servicio de ingreso de procedencia de éste, el tipo de ingresos (programado, de urgencia, de otro servicio hospitalario, programado de consultas externas del departamento, mediante derivación de otro centro hospitalario), la fecha de alta de todos los pacientes, así como circunstancias que concurren al alta y el servicio que proporciona esta.

En cuanto a los datos clínicos estos se obtienen del análisis del informe de alta. De los datos clínicos, los más relevantes serán: diagnóstico principal y secundario.

Plan de análisis. La base de datos proporcionada por la CMBD será analizada con Microsoft Excel y por el paquete estadístico SPSS 19. Se pretende agrupar las variables para poderlas estudiar entre ellas y compararlas por periodos de tiempo.

\section{Resultados}

En lo que se refiere al perfil del paciente que ingresa en la unidad en cada año, basándonos en los datos analizados, podemos decir que:

En el año 2011, el perfil del paciente ingresado fue: hombre $(55,71 \%)$, de entre 41 y 50 años $(29,05 \%)$, cuyo motivo de ingreso es descompensación psicótica $(36,67 \%)$ y con diagnóstico principal esquizofrenia $(24,76 \%)$ que permanece en la unidad entre 8 y 14 días $(26,19 \%)$. Por otro lado, en el año 2012, el perfil quedaría de la siguiente forma: hombre $(50,97 \%)$, de entre 41 y 50 años $(31,55 \%)$, cuyo motivo de ingreso es descompensación Psicótica $(45,15 \%)$ y con diagnóstico principal de trastorno bipolar $(23,30 \%)$ que permanece en la unidad de 8 a 14 días (24,76\%). En cuanto al 2013, observamos que el perfil sigue la misma línea del año anterior, siendo en este año: hombre $(52,63 \%)$, de 31 a 40 años $(27,53 \%)$, cuyo motivo de ingreso es descompensación psicótica $(37,65 \%)$ y con diagnóstico principal de trastorno bipolar $(27,13 \%)$ que permanece en la unidad de 8 a 14 días $(23,48 \%)$. Siguiendo con el análisis de los datos extraídos, vamos ahora a detallar cada variable:

Sexo. Tal y como se observa en la tabla y gráfico siguiente es el sexo masculino el que predomina en los tres años del estudio, aunque podemos apreciar que con el paso de los años la diferencia entre ellos es cada vez menor.

Grupos de edad. En cuanto a la edad, la mayor parte de ingresos corresponden a pacientes con edades comprendidas entre 31 y 50 años en los tres períodos de tiempo estudiados.

Motivo de ingreso. Tal y como se observa en el gráfico (página siguiente), el motivo de ingreso principal corresponde en los tres años a descompensación psicótica. Aunque cabe destacar el incremento importante que existe con el motivo de ingreso autolisis que se ve aumentado en el último año con respecto a los dos anteriores.

Diagnóstico principal. En cuanto al diagnóstico principal, observamos que en 2011 prevalece la esquizofrenia, mientras que en los siguientes dos años 20122013 obtenemos como diagnóstico principal el trastorno bipolar. Observamos también un aumento progresivo del trastorno de la personalidad. 

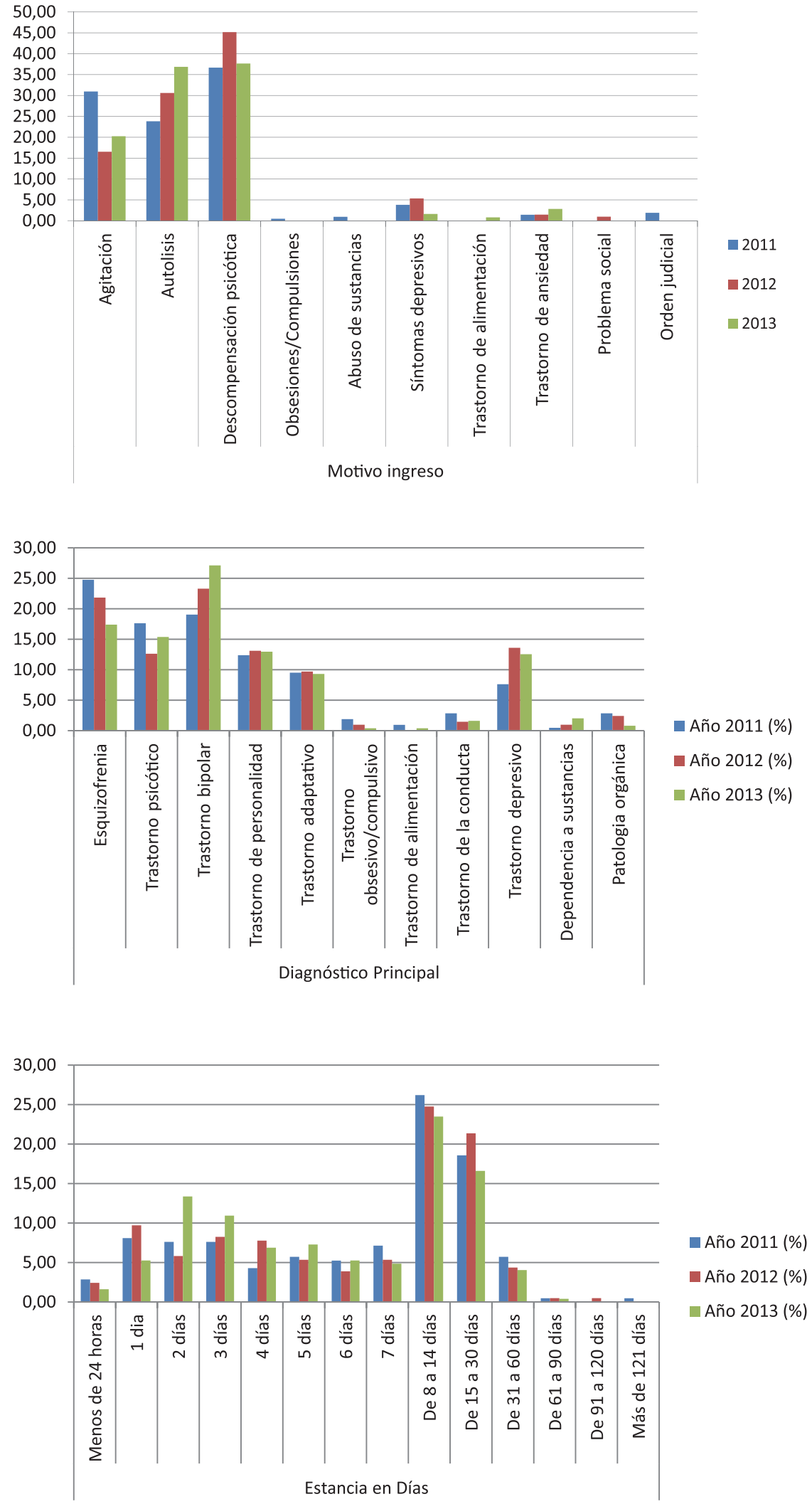
Estancia en días. En cuanto a los días que los enfermos permanecen ingresados en la unidad, se observa que el rango predominante durante todo el período del estudio es de entre 8 y 14 días, seguido de cerca por el rango de 15-30 días.

\section{Discusión}

Tras varios años de trabajo en la UHP del Hospital Comarcal de Gandía, no tener constancia de ningún trabajo de investigación en donde se analice y determine el perfil del paciente que ingresa en dicha unidad, y debido a las características propias de la misma (se trata de una unidad abierta y combinada con Medicina Interna), vemos necesario la descripción del perfil de dicho paciente, para así poder adaptar mejor los objetivos de la unidad a las necesidades del paciente según sus características. Tras el análisis de las variables, podemos concluir que el perfil del paciente que ingresa en ésta unidad es el de un varón de entre 31 y 50 años cuyo motivo de ingreso es la descompensación psicótica, seguido en 2013 por la autolisis; con diagnóstico principal de esquizofrenia, seguido por trastorno bipolar en 2011, mientras que en el 2012 y 2013 predomina el trastorno bipolar seguido de la esquizofrenia; y con una estancia de entre 8 y 30 días de duración.

\section{Bibliografía}

1. Características de los Usuarios de una Unidad de Agudos de Salud Mental: Determinantes Psicosociales y Clínicos, Ramos López, A; Díaz Palarea, M.D. (Vol. XXV, nº 96, Octubre/diciembre 2005, páginas 37-55; Revista de la AEN).

2. PO-382. Distribución por GRD de los ingresos en la Unidad de Hospitalización de agudos de psiquiatría del área sanitaria de Santiago de Compostela en el período 2009-2013. Diéguez Quintairos, A.; Del Río Casanova, L; Brenlla González, J; Blanco Martínez, C; González Castroagudin, R; Pampín Alfonso, A; López De Abajo Rodríguez, B. (XVIII Congreso Nacional de Psiquiatría 2015, Santiago de Compostela).

3. PO-579. Estudio Descriptivo de pacientes ingresados en la Unidad de Hospitalización Psiquiátrica del Complejo Hospitalario Universitario de A Coruña. Diagnóstico y Tratamiento. Vicente Alba, J. (XV Congreso Nacional de Psiquiatría).

4. PO-311. Estudio descriptivo de los pacientes ingresados en una Unidad de Hospitalización de Salud Mental. Rodríguez Jiménez, E; Chauca Chauca, G.M.; Ordovás González, M.P. (XVII Congreso Nacional de Psiquiatría).

5. World Health Organization, The World health report 2001: new understanding, new hope, Geneva, World Health Organization, 2001.

6. Manual Diagnóstico y Estadístico de los Trastornos Mentales, Barcelona, Masson, 1995.

7. DSM-5, American Psychiatric Association, editorial medica Panamericana. 